\title{
LA INTERVENCION ADMINISTRATIVA EN LOS CENTROS URBANOS DE LAS GRANDES CIUDADES
}

\author{
Manuel Castells \\ (Ecole d'Hautes Études en Sciences Sociales, París)
}

La crisis urbana tiene dimensiones múltiples. Como la centralidad resume y sintetiza las tendencias profundas del sistema urbano, la crisis de la centralidad en las áreas metropolitanas condensa de forma particu. larmente intensa las actuales contradicciones del proceso de urbanización. EI Estado, en sus distintos niveles, interviene de forma cada vez más sistemática en el tratamiento de las distintas dimensiones de la crisis urbana. Y por tanto, en todos los países, la acción administrativa ha contemplado con particular interés la renovación de los centros de las ciudades como una forma de revitalizar los tejiclos urbanos y de contrarrestar las tendencias a la deterioración de las ciudades. Ahora bien, en la medida en que la Administración está fuertemente sometida a tensiones sociales y políticas, los procesos de renovación urbana expresan a la vez la dinámica de la ciudad, la dinámica de los grupos sociales, y la dinámica del Estado. Basándonos en las investigaciones llevádas a cabo por nosotros mismos en Francia y Estados Unidos, así como en la literatura urbanística existente sobre el tema, examinamos aquí en qué consiste la crisis de los centros, cuáles son las determinantes sociales de la intervención del Estado, y en qué medida los programas planteados para la renovación de las ciudades tienen posibilidad de alcanzar los objetivos que se asigtan. 
El problema de los centros proviene del inpacto en la estructura de la ciudad, del desfase existente entre los ritmos de evolución del tiempo y del espacio. Es decir, una evolución espacial lenta, cristalizada en formas, y una evolución temporal rápida, sometida al cambio histótico-social. Lo cual quiere decir que una misma estructura social va a articularse diferencialmente a distintas estructuras espaciales. $O$, en términos más urbanísticos, que una estructura espacial va a constituir el mismo soporte físico para distintas funciones urbanas. Sobre esta contradicción entre un espacio cristalizado y un tiempo cambiante, que lleva a un cambio de funciones utbanas en una misma estructura física, se organiza la trama de intereses sociales que tratan de resolver dicha contradicción en un sentido que favorezca a cada uno de esos grupos de intereses sociales, a veces clases, a veces fracciones de clase, a veces alianzas y conglomerados que se pueden consolidar como grupos de presión.

La complejidad del proceso (por un lado esta contradicción y por otro el tratamiento social de la contradicción) requiere la intervención del Estado, como instancia que tiende a tratar los problemas de la sociedad en su globalidad; es decir, superando una contradicción particular, superando intereses específicos, el Estado va a atender, a englobar en su acción los distintos intereses y los distintos problemas. De esta forma puede decirse que los procesos de renovación utbana van a articularse directa o indirectamente, siempre con la acción y los intereses del Estado. $Y$ aquí empieza el problema en cierto sentido, a saber, que esa intervención del Estado no va a ser neutra socialmente, sino que esa intervención va a estar determinada por los conflictos políticos y los grupos de intereses que van a subyacer esta acción del Estado.

Este es el conjunto de problemas que quisiera abordar. En primer lugar tratar de situar cuál es la raíz estructural de los problemas planteados por la centralidad urbana. En segundo lugar ver cómo esos problemas son articulados a una serie de intereses sociales. En tercer lugar, ver cómo el Estado interviene en esa trama de intereses sociales, con respecto 
a esos problemas utbanísticos. En fin, trataré de sugerir algunas características específicas de la crisis de la centralidad urbana en España y particularmente en Madrid.

\section{La crisis de la centralidad urbana}

El planteamiento referente a la crisis de los centros urbanos debe partir del análisis del proceso de formación de la centralidad urbana. La centralidad urbana proviene, en un primer punto, de la expresión a nivel de espacio de lo que los estudiosos llaman desde hace tiempo la división social del espacio. Es decir, en la medida en que hay una división del trabajo en la sociedad, en la medida en que hay distintas actividades y distintos niveles sociales ligados a estas actividades, esta división se espacializa y al espacializarse hay, a la vez, elementos de diferenciación y elementos de coordinación, tanto a nivel social como espacial. ${ }^{1}$

Los centros urbanos son la expresión de esta necesaria coordinación de las actividades y categorías sociales en su dimensión espacial. Es decir, los centros urbanos son la organización espacial de la puesta en relación, del intercambio, de la coordinación, con respecto al proceso de la división social del trabajo.

Un segundo elemento que forma la centralidad urbana es aquel que resulta sobre este proceso de división social del trabajo, de la concentración de actividades, de tecursos económicos y del poder de decisión tanto en lo público como en lo privado. Es decir, no solamente hay una división social que coincide en la división espacial y, por tanto, en la coordinación, sino además esta división de tareas no es simétrica: es una división de tareas que conlleva una serie de procesos de concentración tanto de actividades como de poder, lo cual se expresa en una centralización de las actividades direccionales que se añaden y se articulan a las actividades de coordinación y de intercambio.

Se asiste, pues, a un doble proceso; por un lado, la concentración creciente en unas ciudades de las actividades sociales y económicas; la concentración a su vez, dentro de estas ciudades, de dichas actividades en su nivel direccional, en lo que se llaman los centros urbanos. Primer elemento: coordinación; segundo elemento: concentración de actividades; tercer elemento: se relaciona a la necesaria accesibilidad de las zonas cen.

1. Sobre este tema, véanse los planteamientos que hicimos hace tiempo en nuestro artículo «Le Centre urbain», Cabiers Internationaux de Sociologie, 1 (1969). 
trales, conforme se desarrolla el proceso de crecimiento urbano. Es decir, cuanto más crecen las ciudades, más necesaria es una jerarquización de los servicios, y por tanto, una accesibilidad espacial que tiende a concentrar estos procesos de accesibilidad en las zonas centrales, que van a ser zonas no determinadas en términos de centralidad geográfica sino en términos de accesibilidad con relación del sistema de transportes. ${ }^{2}$

Un cuarto proceso que concurre a la formación de la centralidad es la difetenciación simbólica del espacio y la concentración de dichos procesos simbólicos en ciertos lugares espaciales. Es decir, todo el espacio está cargado de significación pero además, como hay una jetatquía social implícita en esta valoración simbólica, va a haber también una jerarquía de la organización espacial. La centralidad urbana va a ser, por tanto, también la expresión del nivel más alto de esa jerarquía simbólica. ${ }^{3}$

A partir de estos cuatro elementos: división social del trabajo, concentración de poder, accesibilidad ligada al crecimiento urbano y jerarquización simbólica, se constituye una expresión de la estructura espacial en las grandes ciudades, en términos del valor que recibe el suelo en el mercado urbano; es decir, estos cuatro criterios se combinan para expresarse en una sintesis que, en nuestro tipo de sociedades, se expresa por los precios del suelo. Estos precios del suelo son los que van a expresar la centralidad y la periferia urbana.

Una vez estos precios del suelo dan lugar a una organización de la localización de actividades, siguiendo estos criterios producidos económicamente, entonces se produce un reconocimiento y una institucionalización de esta estructura urbana y de esta estructura espacial por parte de las normas administrativas. Así pues, a un primer nivel, hay una serie de relaciones entre la estructura social y la estructura espacial. A un segundo nivel, la traducción de esto en nuestras sociedades es el sistema de precios del mercado. A un tercer nivel, la cristalización de estas determinaciones sociales y económicas se hace mediante normas administrativas y por tanto mediante la organización propiamente jurídico-política que entonces organiza, refuerza, institucionaliza esta centralidad urbana así expresada.

¿Por qué hay crisis en este sistema de centralismo? Porque el cam. bio permanente en que se encuentra la sociedad produce cambios en la estructura social, cambios que asignan a una ciudad, por tanto, a sus

2. Véase K. H. Schaeffer y Elliot Sclar, Access for All: Transportation and Urban Growtb (Londres: Penguin Books, 1975). $1960)$.

3. Vease Kevin Lynch, The Imoge of the City (Cambridge, Mass.: MIT Press, 
centros, nuevas funciones urbanas. Estas nuevas funciones urbanas chocan con la rigidez de las formas espaciales existentes, produciéndose un desfase entre la permanencia de las formas espaciales y la novedad de los usos sociales. Uno de los aspectos de ese desfase es el aspecto funcional, en el sentido ya conocido de la saturación de actividades que hace que en un centro ya construido, se continúen localizando actividades, puesto que esta aparición de actividades centrales está ligada a los procesos que ya hemos señalado anteriormente de concentración de poder y de intensificación de la división de trabajo. Estas actividades nuevas necesitan una ligazón al espacio central; espacio central que ya está saturado por la construcción física preexistente.

Otro ejemplo típico de esto, quizá el más claro, son las funciones circulatorias en el sentido de que los nuevos flujos urbanos que se pro ducen, los nuevos medios de transporte, en particular en el caso del automóvil, determinan un uso de la viabilidad totaimente desfasado con respecto a las estructuras físicas, por ejemplo, en nuestro país, muy frecuentemente ligadas a todo un pasado medieval. En este sentido hay un desfase muy claro entre el nuevo uso exigible y las nuevas formas espaciales existentes. Pero esta adaptación no es sólo funcional; la adaptación funcional, de hecho, sirve de soporte a otros procesos mucho más complejos y mucho más contradictorios. En términos puramente físicos cualquier urbanista sabe que se puede respetar una ciudad medieval introduciendo el autom6vil en los flujos urbanos y en los medios circulatorios, pero tomando toda una serie de medidas preservadoras con respecto al uso del automovil.

Pero el problema es que esta adaptación funcional, en general, sirve de eje de penetración a otros aspectos mucho más dominantes. A saber: la adaptación necesaria en el nuevo proceso de valoración del suelo. La dominación del valor del cambio sobre el valor de uso en nuestra economía, hace que las nuevas funciones urbanas valotizadas, a saber, la implantación de funciones direccionales, la residencia de categorías sociales elevadas, en términos de ingresos y estatus social, tiendan a desplazar a las actividades y a la población existentes por tradición histórica en ese espacio.

El espacio central pasa a ser altamente valorizado en términos de precios, en términos de mercado utbano; pero al mismo tiempo esa valoración es potencial, no puede realizarse, puesto que hay una rigidez de la ocupación existente. Desfase, pues, entre Ia débil rentabilidad económica para el capital privado del uso existente y la alta rentabilidad potencial para el capital de los nuevos usos, a los cuales tendería la estructura urbana en su dimensión central. De aquí el hecho de que se tienda entonces a adaptar los 
valores de uso a los valores de cambio. A un tercer nivel se plantea otro desfase: la deteriorización de edificios y condiciones de vida, de equipamiento urbano en el centro con respecto a los estándares de equipamiento y de vivienda a los que llega la sociedad, por envejecimiento de las es. tructuras físicas que existen en los centros antiguos que, en general, son centros de una edad urbanística mayor que el resto, por una razón elemental: toda ciudad crece del centro a la periferia.

Esta deteriorización de servicios y niveles de equipamiento urbano es una consecuencia de los procesos antetiores y no la determinante de la renovación; es decir, lo que se va a utilizar como justificación pública de una operación de renovación, que es el envejecimiento físico de las estructuras no es sino la consecuencia de los otros procesos. Es por la expectativa de sustitución de usos y por la dimensión económica y funcional de esta expectativa por lo que hay deterioro, y no teparación de las estructuras existentes.

Es decir, toda estructura física puede mantenerse si hay una política, tanto pública como privada, de conservación de estas estructuras; si no se mantienen es porque los intereses funcionales y los intereses económicos subyacentes no representan un estímulo suficiente para ese mantenimiento físico de las estructuras.

Aquí introduzco una idea que desarrollaté más adelante en las alternativas, a saber: Que una de las mayores contradicciones del desarrollo urbano es que se realiza por destrucción de las estructuras existentes y adición de nuevas estructuras físicas, cuando es evidente que una política centrada en el patrimonio urbano existente debería más bien partir de la conservación y mantenimiento de todas las estructuras existentes y des. pués de añadir para las nuevas necesidades. De hecho, nuestras ciudades progresan autodestruyéndose en lugar de ir creciendo a partir de una base de mantenimiento de lo ya existente.

Este tema de política urbana es uno de: los elementos que están apareciendo en los últimos diez años en todas las polémicas respecto al nuevo modelo de crecimiento uxbano: Crecer en términos de crecer sobre lo construido y no crecer en términos de añadir barrios periféricos sin fin y dejar destruirse las ciudades ya existentes.

Este proceso de adaptación funcional, adaptación física y adaptación de rentabilidad económica de los usos del suelo central es un proceso organizado, trabajado, por los intereses económicos y sociales dominantes. En este sentido, sobre estos tres elementos que empujan hacia una teno. vación urbana, va a producirse un doble movimiento de grupos de intereses que van a intentar plantear la necesidad de una renovación. Por un lado, las empresas y administraciones que necesitan una adaptación fun- 
cional del centro para la implantación de actividades direccionales y para la organización de los intercambios, tanto de personas, como de metcancías, necesarios al crecimiento urbano; es decir, va a haber una serie de intereses funcionales en que esta renovación se realice. Por otro lado, va a haber unos intereses del capital del sector inmobiliario en controlar y dirigir esta operación, en la medida en que la producción y comercialización de bienes urbanos, ligados a una sitzración central, es, en general, la que produce una más alta rentabilidad, una más alta tasa de ganancia. Con una condición: con la condición de que se creen previamente las condiciones de rentabilidad para implantar nuevas actividades. Es decir, que esa rentabilidad del capital privado va a ser posibie si previamente hay una puesta en condiciones del suelo central para la producción de esos equipos urbanos. En esas condiciones la más alta tasa de ganancia está ligada al desarrollo de actividades direccionales.

De ahí es absolutamente necesario que esta renovación de los centros sea organizada y cubierta por el Estado, por la Administración, en sus distintos niveles, Central, Provincial o Local, fundamentalmente, por tres razones. En primer lugar, para superar el fraccionamiento de la propiedad del suelo y para crear mecanismos que permitan reagrupar convenientemente la propiedad del suelo o proteger un reagrupamiento privado de la propiedad del suelo, de manera que dicho reagrupamiento haga operaciones de escala rentable. A un segundo nivel, la razón fundamental de la intervención del Estado es la necesidad de financiar los costes de demolición, desplazamiento y creación de infraestructura urbana necesaria a la puesta en condiciones del suelo central, para asignarles nuevos usos. En tercer lugar, dado el costo social de toda operación de renovación urbana, debido al desplazamiento de actividades y de población, es necesaria la intervención de los entes públicos para legitimar socialmente la destrucción del patrimonio y el desplazamiento de la población en aras del interés general.

Desde luego, no toda la renovación urbana es de iniciativa estatal. Pero el Estado interviene de forma decisiva, directa o indirectamente, para abriz brecha en un casco urbano; y a partir de un primer proceso de renovación, entonces, hay difusión de estos efectos, tanto en términos de rentabilidad como en términos de legitimidad social, a zonas nuevas del casco urbano, pero que han sido primero introducidas por una politica dominante pública.

En resumen, a la crisis del centro responde la adaptación funcionai, económica y física necesarias a esta crisis del centro. Pero al mismo tiem. po se utiliza esta crisis del centro y las necesarias operaciones de adap- 
tación, por el capital inmobiliario y por los intereses funcionales de las empresas. Sobre esta trama de relaciones se produce la intervención del Estado.

Ahora bien, el Estado no es un agente: neutro; el Estado no es tampoco un instrumento de una minoría oligárquica como ciertas visiones esquemáticas, a veces, tratan de señalar. La intervención del Estado es una intervención compleja, determinada en el plano del urbanismo, en primer lugat, por los conflictos políticos y sociales que subyacen a la acción pública, es decir, por el enfrentamiento en el seno del Estado y con respecto al Estado de grupos sociales, de grupos de intereses. ${ }^{4}$

La acción del Estado va a venir determinada también por la lógica del funcionamiento interno $\mathrm{y}$, por tanto, por el peso histótico, otganizado institucionalmente, que ha condicionado a to largo de la historia la formación del Estado tal y como es. Por tanto, por su propia lógica administrativa, que es producto de la presión y conservación de la historia social.

En el caso del urbanismo, la intervención estatal también setá condicionada por los modelos urbanísticos imperantes en los agentes de intervención pública; modelos urbanísticos que, evidentemente, van a provenir de la sociedad, pero teniendo en cuenta la autonomía propia que ciertos modelos urbanísticos tienen en los entes públicos con respecto a su determinación general por los intereses sociales.

Es decir, el proceso de intervención del Estado es determinado a la vez por condicionamientos sociales y políticos, por el funcionamiento de la estructura interna del Estado y por los modelos culturales y utbanísticos que utiliza la Adrainistración.

Así pues, to que se presenta como una intervención coordinadora, racionalizadora, planificadora, se convierte en un proceso social, político y cultural. Por tanto, los determinantes de este proceso van a ser decisivos en el contenido de la intervención urbanística y, por tanto, en el futuro de los centros de las ciudades.

\section{Experiencias internacionales de renovación urbana}

Trataremos de conectar la problemática expuesta a partir de los resultados de investigaciones sociológicas sobre el tema. De forma directa hemos realizado dos investigaciones detalladas sobre esta cuestión: una

4. Véanse los análisis reunidos en el libro colectivo dirigido por Nicos Poulantzas, La crise de l'Etat (Parf́s: PUF, 1976). 
sobre la renovación del Centro de París ${ }^{5}$ y otra más global sobre las tendencias generales de la renovación urbana en las ciudades norteamericanas. ${ }^{6}$ En el caso del centro de París, que ha sido uno de los programas más brutales que se conocen, en el que hubo demolición masiva de toda una parte del centro, la investigación que nosotros hicimos, al principio de haberse planteado el programa, se basó en un método muy simple: en comparar las características físicas, funcionales y sociales del espacio existente en los sectores que iban a ser renovados, con las catacterísticas tesultantes de esos sectores si los programas de renovación, fueran o hubieran sido completamente cumplidos. Método absolutamente elemental, simplemente con problemas de informacion, pero el método es bastante claro y simple. Sin entrar en la metodología, que se expone en las fuentes citadas, los resultados más importantes son los siguientes: Se probó que en la renovación dei centro de París, toda la ideología justificadora, en términos de eliminar los tugurios, era absolutamente infundada. En el sentido de que en primer lugar los sectores más deteriorados de la ciudad de París no exan aquellos que eran objeto de programas de renovación. Había sectores muy deteriorados que no iban a ser renovados. Es decir, todos los sectores renovados eran sectores deteriorados, pero no todos los sectores deteriorados etan renovados.

Por otra parte, las variables más importantes en provocar el proceso de tenovación eran: en primer lugar, las variables de carácter social: se renovaban, sobre todo, aquellos barrios de extracción muy obrera y en particular los que tenían alta proporción de trabajadores inmigrados. En segundo lugar, se renovaban aquellos barrios en los que había una necesidad funcional de implantación de oficinas y de actividades direccionales. En texcer lugat — sólo en tercer lugar - venían las variables de tipoestado de la vivienda, deterioro físico de la estructura urbana, equipamiento, etc. Más aún, nosotros probamos que a nivel de equipamiento se mantenían las mismas, antes y después; que lo que sí se producía era una sustitución de usos funcionales y una sustitución de población. Es decir, se liquidaban actividades industriales, pequeños talleres, pequeños comerciantes, y se implantaban grandes centros direccionales de oficinas, supermercados y comercios de lujo. En términos de población se elimi-

5. Véanse los ptincipales resultados de la investigación expuestos en mi attículo «Urban Renewal and Social Conflict in París», Social Sciences Intormation (agosto 1972).

6. Véanse los dos análisis realizados sobre el tema: «La ténovation urbaine aux Etats-Unis», Espaces et Sociêtés (noviembre 1970). Y sobre todo la Parte V, dedicada al estudio de la crisis urbana en los Estados Unidos, de nuestro libro Tbe Urban Question (Cambridge, Mass.: MIT Press, 1977). 
naba la población obrera, de bajos ingresos, y se pasaba a construir residencias de alto nivel social, en general, técnicos superiores y profesionales. Un proceso bastante clásico; simplemente que era lo contrario de lo que se proclamaba en la ideología urbanística oficial, por lo cual hubo momentos de escándalo público como resultado de estas investigaciones.

El otro elemento que nosotros señalamos cuando hicimos la investigación, hace ya tiempo, en el año 1970, fue que había un fuerte impacto político de la renovación. Un impacto político en el sentido de que se desorganizaban barrios populares, con alto porcentaje de votos de izquierdas, con una relativa implantación de los partidos de izquierda y que todo el proceso iba hacia una radicalización, en términos políticos, bacia la detecha, y en particular hacia el Partido Gaullista, en base a un doble meca* nismo: por un lado, la población que allí se iba a implantar; en otro aspecto, en base a los mecanismos de gestión administrativa que se iban situando en los barrios de París, conforme avanzaban los procesos de renovación.

Nosotros planteamos como hipótesis en el año 1970, que uno de los problemas políticos del partido gobernante en Francia era su falta de implantación a nivel local. Una de las hipótesis de política general de las democracias occidentales es que un partido sólo se conserva durante largo tiempo en el poder cuando la implantación local asegura el servicio cotidiano de la población. ${ }^{7}$ Este ha sido el Talón de Aquiles del Gaullismo en Francia; al no haber podido asegurar nunca una representación popular estable. Sea por la izq̨uierda o por la detecha, a excepción de Burdeos, todas las ciudades francesas se escaparon siempre al control directo del aparato del Partido Gaullista.

Nuestra hipótesis fue que en el caso de París se ha aprovechado el hecho de que París era la única ciudad -exceptuando España y Pottugal- que en aquellos momentos no tenía ninguna autonomía popular, por una razón histórica muy sencilla: la Comuna de París. Desde 1871 París era una ciudad directamente gestionada por el Primer Ministro. Nuestra hipótesis fue que una vez el ptoceso de cambio social y político en París estuviese asegurado en parte, con cargo al programa de renovación urbana, se crearía una nueva Ley de Autonomía Local y se eligiría un alcalde de París que sería la expresión, a nivel local, del Partido Gaulista. Si hoy insisto en esta hipótesis es porque lo escribí claramente en el año 1970 y fui acusado de demagogia política en aquel momento. Parece que no lo era tanto: era simplemente observar los resultados po-

7. Véase, para una discusión de estos temas, nucstro trabajo «La cuestion municipal», Argumentos (junio 1978). 
líticos que se iban produciendo en los barrios de París conforme avanzaba la renovación.

Aś́, pues, se observa la articulación, en el caso de la renovación parisina, de tres determinantes en el proceso de renovación: la determinante del cambio funcional necesario a la ciudad de París para convertirse en un centro direccional, correspondiente a la nueva otganización de la región metropolitana de París. Un segundo aspecto son las considerables ganancias ofrecidas al sector privado por esta operación de renovación en base al sistema de empresas mixtas que organizaban la operación de renovación, de forma que los fondos públicos aseguraban los gastos de demolición y saneamiento del terreno. Las prerrogativas públicas aseguraban la movilización de la propiedad urbana, en el sentido expropiatorio, por razones de utilidad pública, asegurando al capital privado la promoción rentable de los nuevos edificios y servicios a construir en el centro de París. A un tercer nivel, junto a la utilidad funcional y la utilidad económica para los sectores de capital privado, una utilidad política para el aparato del Estado, controlado en su más alto nivel por el Partido Gaullista; por tanto, articulación de intereses funcionales, económicos y políticos en el tratamiento del espacio urbano por medio del programa de renovación del centro.

De manera mucho más resumida aún expondremos una hipótesis setmejarte para el caso de la renovación de las grandes ciudades rorteamericanas. El programa de renovación trbana en los Estados Unidos, que se empezó en la práctica en el año 1962-1963 y duró hasta 1975, es quizás el programa urbano más gigantesco de la historia de la humanidad en términos de recursos movilizados y de efectos producidos sobre la estructura urbana, con cerca de cuatro millones de personas desplazadas. Basándonos en los datos existentes, demostramos que había tres características fundamentales de este programa. En primer lugar, era un programa absolutamente reducido al centro de negocios de las ciudades, de las grandes ciudades y, en absoluto, a las zonas más deterioradas que eran las zonas que en el urbanismo norteamericano se llaman ciudad central. Lo realmente deteriorado es la ciudad central y no el centro urbano y lo que realmente se renovó fue el centro urbano y no la ciudad central.

Por otra parte, estos programas fueron dirigidos a erradicar poblaciones de minorías étnicas, ya que el $70 \%$ de las personas erradicadas por la renovación urbana norteamericana eran personas pertenecientes a alguna minoría étnica, es decir, negros, puertorriqueños, mexicanos, chinos, japoneses, etc. ${ }^{8}$ Por consiguiente, el programa actuó como una especie de cordón

8. Particularmente importantes en este sentido son las investigaciones de Chester Hartman. 
sanitario que amenazaba con su presencia física inmediata el orden social y el orden público de los centros direccionales existentes en el centro de las ciudades norteamericanas.

La tercera característica fue que esta tenovación impalsó, sobre todo, dos tipos de actividades: por un lado, la preservación y expansión de los grandes centros de negocios; por otro lado, la preservación de ciertas instituciones culturales de élite, cuya ubicación central exa absolutamente necesaria. Por ejemplo, uno de los casos más importantes en la renovación norteamericana es el de las grandes universidades. Las grandes universidades, implantadas en los centros de las ciudades a mediados y finales del siglo XIX, se vieron rodeadas por los ghettos, con el consiguiente deterioro del prestigio y del nivel de los barrios citcundantes.

El programa de renovación urbana, tanto en los Estados Unidos como en París, según demuestran los estudios realizados por nosotros mismos, es un programa con un contenido clasista, muy explícito, incliuso consciente, y en los dos casos -incluso en los Estados Unidos también- la intervención del Gobierno, al nivel más alto, es fundamental. En los Estados Unidos es el Gobierno Federal, hay en particular progtamas muy centralizados en Washington $D C$, en los que se proveen dos tercios de los fondos públicos para los municipios y en los que se articulan realmente los intereses politicos con los intereses económicos y con los intereses funcionales, sociales y culturales de preservación de la élite.

En lo que se refiere a Madrid, fundándonos en los estudios que empiezan a existir, en particular sobre el libro de Alfonso Alvarez Mora, sobre la remodelación del centro de Madrid, y una tesis doctoral de Rafael Mas sobre los cien años de historia del Barrio de Salamanca, los datos de que disponemos indican que la renovación de Madrid es más brutal y más directamente ligada a los intereses de grupos financieros monopolistas y sin ninguna de las protecciones que en el caso de París o en el de los Estados Unidos sí existían, en cierto sentido, ni de la población, ni del patrimonio urbano, aprovechando la situación política existente de falta de libertades y de falta de representación política a cualquier nivel, combinada con un urbanismo de «autopistas», desembocando en lo que casi se podría calificar de "Urbanismo de horca y cuchillo»."

Este proceso de París, de los Estados Unidos, o de Madrid, no es un proceso ineluctable $y$, desde luego, no es aceptada la tesis según la cual es la necesidad inherente a todo desarrollo urbano capitalista. Evidentemente hay intereses capitalistas. Pero el capitalismo no es sólo el capital

9. Alfonso Alvarez Mora, La remodelacion del centro de Madrid (Madrid: Ayuso, 1978). 
y en una sociedad capitalista suceden muchas más cosas que las impulsadas por los grupos capitalistas y la especulación del suelo urbano. Incluso en una sociedad capitalista puede haber fenómenos de renovación urbana de signo muy distinto.

Es bien conocido el caso de Bolonia y, por tanto, no se trata de añadir ningún cartel propagandístico más a los conocimientos sobte este tema, pero se trata de una demostración palpable de lo afirmado." Incluso en otros contextos (como el caso del Barrio de Marolies, en Bruselas) en el que la renovación de un barrio popular se planteaba estrictamente en los mismos términos que la de París, se llega a resultados muy distintos tras una presión popular importante. En efecto, se creó un comité de preservación, cuyo presidente era el príncipe Alberto de Lieja, que impulsó una movilización popular y ciudadana, ligada a grupos de élite cultural que se opusieron al proyecto, obteniendo una renovación social, restaurando el contenido social y populat del barrio, haciendo un barrio preservado históricamente y preservado en su contenido de vida social y, además, con la participación de la población en los programas de remodelación, de forma que sirvió luego para extenderlos a otras experiencias belgas. ${ }^{11}$

También es verdad que incluso en el mismo contexto belga todo depende de los intereses que estén en juego, porque, al mismo tiempo, en la tenovación del Norte de Bruselas - el proyecto llamado Manhattan-, como se trataba de hacer un centro direccional para las multinacionales implantadas en Bruselas, no se aceptó la participación ciudadana y se ims. pulsó el proyecto a golpe de bulldozer. ${ }^{12}$

En otro contexto puede citarse la experiencia del batrio de Christiania en Copenhague, que era una isla-parque en el centro de la ciudad, ocupada por instalaciones militares. ${ }^{33}$ En el año 1971 el Ejército danés cedió sus terrenos del centro de la isla al Ayuntamiento de Copenhague, para que hiciera el uso público que estimara conveniente. Se trataba de una ocasión absolutamente extraordinaria, con un parque, un lago y viejos edificios de valor histórico. Mientras la ciudad de Copenhague pensaba qué hacer con aquella maravilla y cuando ya había un proyecto de grandes centros financieros, un grupo de unos setecientos ecologistas libertarios ocuparon ese

10. Véase P. L. Cervellati y R. Seannavini, Bologna: Politica e metodologia del restauro net centri storici (Bolonia: It Mulino, 1973).

11. Nos hemos fundado en la investigación que sobre el tema lieva a cabo, bajo nuestra dírección, Cecilia Añaños, en la Escuela de Ailos Estudios de París. Melis.

12. Según los trabajos que hemos dirigido, de Michel Hengchen y Christian

13. Encuesta directa realizada durante mi estancia como profesor-visitante en la Escuela de Arquitectura de Copenhague en matzo de 1976. 
centro y lo declararon la "Comuna Libre de Christiania», con gran escán" dalo del capital inmobiliario y de algunos sectores de la comuna de Copenhague. Pero la opinión pública se movilizó porque era interesante ver cómo organizaban la comuna y los domingos la gente empezó a ir a ver esas formas alternativas de vida. En fin, el Ayuntamiento de Copenhague decidió declarar Christiania experimento social durante cinco años y enviaton una legión de sociólogos y psicólogos para que lo estudiatan. Se obtuvieton algunos resultados en términos de tratamientos de droga: el $25 \%$ de la población exa drogadicta y el modo de vida de Christiania demostró ser de gran eficacia para su recuperación. En el año 1976, en que se acabó el plazo de los cinco años, se intentó recuperar el ritmo normal de la reno. vación urbana. Les ofrecieron a los ecologistas fundar una comuna en un terreno regalado por el Estado en el norte de la península de Jutlandia, pero ellos rehusaron porque su comuna, de hecho, eta parte indisoluble de Copenhague. Hubo una campaña pública que detendió y preservó esta experiencia, por to que se ha convertido en un centro de vida cultural y artís. tica, en un centro de emisión de nuevas idests, por muy discutibles que puedan ser.

De este conjunto de expetiencias puede deducirse la diversidad de las vías sociales de tratamiento del problema de la centralidad. Por eso nos mostramos en desacuerdo con el hecho de que, a veces, extrapolando los resultados de nuestras investigaciones, se ha decretado que en un centro urbano todo viene determinado por la voracidad del capital monopolista. En tealidad, hay una diversidad de situaciones y una gama de oportunidades sociales. Incluso el destrozo del centro de Madrid no tiene paralelo en el centro de Barcelona, porque la burguesía catalana ha sabido respetar su ciudad de manera mucho más eficaz que en Madrid. Esto no es por una superioridad racial, sino simplemente porque Madrid ha sido siempre una ciudad artificial creada más bien en base a la burocracia centralista y a la inversión en Madrid del poderío de la oligarquía latifundista y financiera del país, con lo cual ha sido más bient campo de maniobras que una ciudad construida por una clase a través de su historia. La burguesía catalana tiene una historia; la burguesía madrileña la única historia que tiene son las devastaciones de los campos de Castilla. En ese sentido, hay que tener en cuenta que la renovación de los centros depende, fundamentalmente, del proceso social de tratamiento de las contradicciones a las que nos hemos referido, incluso en los dos casos extremos señalados.

En el caso de París, la protesta popular a la brutalidad del programa de renovación urbana de Patís fue determinante; no de manera directa por. que los movimientos de resistencia fueron aplastados violentamente, pero sí en términos de la opinión pública que se suscitó. Por ejemplo, en base a 
nuestras investigaciones hubo una obra de teatro que se montó y que fue representada en el Teatro Nacional Popular, causando bastante impacto sobre la opinión pública en términos de lo que era la renovación urbana; hubo también una setie de campañas de prensa. Se creó tal ambiente que el primer acto de Giscard d'Estaing, cuando asumió la presidencia fue detener la renovación urbana de París, transformando las características de esta renovación, haciéndoia si no más social, al menos sí más cultural, más ligada a la preservación de un patrimonio artístico-histótico y a la defensa de entidades culturales.

En el caso de los Estados Unidos, de hecho, los programas de tenovación son los que han suscitado, por primera vez, los movimientos de reivindicación de base urbana en la mayoría de las grandes ciudades norteamericanas. A partir de ahí, se ha creado tal oposición que hoy en día salvo en Los Angeles, la renovación está prácticamente penalizada, en las gtandes ciudades, hasta el punto de que en Chicago, donde quieren hacer un gran programa de renovación en estos momentos (Chicago 21), la primera medida que ha tomado el nuevo alcalde de Chicago ha sido reunir a las Asociaciones de Vecinos de Chicago para que opinen y organicen el plan de renovación deî norte de Chicago, en unos terrenos ferroviarios, donde no hay que desplazar nada ni a nadie.

En Madrid hay un ejemplo patente de lo que está pasando; la renovación de Malasaña, donde hay una movilización popular, fue detenida. La renovación del casco antiguo de Madrid, la zona de Lavapiés, está siendo, en cierto sentido, modificada en términos de una mayot representación de asociaciones ciudadanas en la tenovación, a partir de la acción de los vecinos de la Asociación «La Corrala». En cambio, en el barrio de Salamanca, donde la representación vecinal es débil y los intereses en juego mucho más poderosos, se está produciendo un rápido proceso de renovación especulativa.

En ese sentido, el cambio de situación, a rivel de modelo político español, a nivel también de crisis económica que implica necesariamente otro tipo de urbanismo menos extensivo y más cualitativo y también el cambio de situación a nivel cultural de lo que la sociedad española hoy día está dispuesta a aceptar o no, permite, quizás, un nuevo modelo de desarrollo urbano. Ese nuevo modelo de desarrollo urbano puede modificar las orientaciones en el planeamiento urbanístico del centro de Madrid o del centro de las ciudades españolas en general. Tratemos de precisar, esquemáticamente, algunas orientaciones en este sentido. 
Del análisis sociológico a la politica urbana: Una alternativa de planeamiento para el centro de Madrid

De los análisis expuestos se deduce el condicionamiento de los procesos de renovación urbana por el modelo social y político predominante. Dado el cambio producido en España en las bases mismas del modelo, se abren nuevas perspectivas a la acción administrativa y a la participación ciudadana. Pero estas condiciones favorables sólo prosperarán en iniciativas fecundas si hay claridad en el diagnóstico y decisión en las medidas urbanísticas urgentes que son necesarias para renovar el centro de nuestras ciudades, y de Madrid en particular, en beneficio de sus habitantes.

Para avanzar en esa perspectiva, partiremos de tres puntos y propondremos diez medidas. Los presupuestos son: en primer lugar, la necesidad de la inserción de toda política de renovación urbanistica de los centros en una politica urbana y regional. No hay posibilidad $-\mathrm{y}$ esto no es simple coherencia cartesiana- de una política de centro sin una política urbana y regional, como veremos al proponer las medidas. En segundo Iugar, la politica relativa al centro debe respetar los intereses de los babitantes de los centros urbanos, pero no debe considerar que el centro es asunto exclu. sivo de esos babitantes, puesto que los usuarios del centro son los habitantes de toda el área metropolitana. Y tercer presupuesto: es fundamental la iniciativa estatal en la renovación urbana, y por estatal nos referimos a la intervención administrativa a todos los niveles, central, regional, provincial y local, pero dicha intervención hay que situarla en el marco de una economía de mercado y, por tanto, con un suelo no socializado. En estos momentos cualquier alternativa urbanística que no parta de estos supues. tos es una hipótesis académica más que una recomendación de poiftica urbana.

A partir de estas bases, ¿cómo abordar el problema de la centralidad de forma más concreta, en términos de medidas de política urbana? El primer tema se relaciona con la problemática de la descentralización regio. nal y de la limitación del creciniento de Madrid. Todo lo que no sea poner límites a este crecimiento y tomat las medidas de política tegional necesarias para esto, significará que toda política urbanística en Madrid, Barcelona o Bilbao será una política de parches, será una política que tratará de tapar vías de agua, y antes de que éstas estén taponadas, nuevas vías se habrán abierto en el casco del núcleo urbano que las grandes ciudades representan. Éste no es el mismo tipo de supuesto que el de la socialización general del suelo. A muy corto plazo, puede abordarse una política de descentralización de actividades y reorganización del poblamiento del territorio que no neces sita grandes medidas de transformación revolucionatia de la sociedad. Pero 
sin estas medidas, la mayor parte de las que proponemos a continuación serían bastante limitadas.

La segunda medida, que sería la traducción a nivel intrametropolitano de la anterior, es la necesidad absoluta de desconcentración de actividades terciarias en el área, dentro del área metropolitana. Es decir, no hay ninguna razón, sino más bien todas las contrarias, salvo la especulación urbana, para seguir concentrando ministerios, oficinas y todas las actividades comerciales en un perímetro urbano central muy delimitado. El razonamiento en términos de accesibilidad está demostrándose en la práctica que no se sostiene, puesto que cada vez hay menos accesibilidad a ese centro en términos físicos. La razón fundamental, de hecho, es la inmovilización de las inversiones inmobiliatias en ese suelo central, y el mantenimiento de un cierto tipo de privilegios en el sistema de transporte para los grandes almacenes, por ejemplo, el condicionamiento del trazado del metro con respecto a la localización de los grandes almacenes. Un Estado que se ocupara seriamente de teorganizat los centros de las ciudades podría tomar otras medidas, como son la desconcentración de actividades administrativas. No hay ninguna razón para que los ministerios de Madrid tengan sus dependencias en el centro. En ese sentido, un buen sistema de transportes periféricos y un ejemplo dado por el Estado de desconcentrar las actividades ministeriales, crearía una posibilidad de un nuevo modelo de desarrollo urbano. Evidentemente, esto no es posible mientras la periferia siga siendo asimilada al destierro. Es decir, las connotaciones siberianas de Ia periferia de Madrid tienen que ser rectificadas en una política de centralidad, que no se ocupe sólo del centro, sino de la descentralización.

$A$ un tercer nivel, es necesario detener el deterioro de las estructuras físicas y, por tanto, lievar a cabo un programa de reconversión y rehabilitación de las viviendas populares deteriotadas en el centro de Madrid. Aquí está el viejo círculo vicioso que casi se hará círculo infernal para los vecinos que lo viven. A saber: por un lado, la falta de rentabilidad de estas viviendas para los propietarios por la Ley de Arrendamientos Urbanos; pot otro lado, el hecho de que el propietatio, como no le es rentable, juega a la ruina y, por tanto, deja que el edificio se deteriore. Es necesario salir de este círculo vicioso con una política urbana que sea audaz y que no tema un cierto grado de impopularidad a corto plazo.

Es necesario hacer una reconversión de la intervención pública en la vivienda particular del Instituto Nacional de la Vivienda, que tienda más, o al menos primordialmente, hacia la preservación del patrimonio urbano existente, como forma de habitación, que hacia la subvención a polígonos periféricos infradotados. Lo cual pasa con una serie de medidas del tipo de obligar a los propietatios a reparar o vender. Reparar con créditos o 
vender al Ayuntamiento o a los inquilinos a precios tasados, pero no leoninos, con subvención en términos de política crediticia a aquellos inquilinos que puedan adquirir su vivienda, o al Ayuntamiento en el caso de que sean inquilinos que no puedan adquirir su vivienda; que pase a ser patrimonio municipal por medio de una transformación de los presupuestos existentes de viviendas sociales.

Un segundo tipo de medida que sería complementaria y no necesaria. mente exclusiva de la anterior es, en ciertos casos limitados, la liberación controlada de los alquiletes. En casos, que pueden ser muy frecuentes, de incapacidad de pagar los alquileres por parte de los inquilinos existentes, subvenciones a estos inquitinos. Que en algunos casos pueden ser del Instituto de la Vivienda o en otros casos, por ejemplo de los jubilados, pueden ser con cargo a la Seguridad Social. En todo caso son medidas que pueden permitir el abordar una politica en que al propietatio se le obligue a reparar, porque obtiene una posibilidad de movilización de esa vivienda en el metcado de alquileres urbanos y así cobra mayor fuerza el control de la acción puramente especulativa de los propietarios.

En tercer lugar, evidentemente, será necesatio en algunos casos una remodelación parcial de viviendas por la Administración, en casos en que efectivamente sean viviendas sumamente deterioradas, en las que no tiene sentido entrar en reparación y que, por tanto, sea necesario hacer operación de remodelación. Y al mismo tiempo, la construcción en terrenos municipales existentes de viviendas sociales que sean de transición, que permitan la teorganización de este conjunto de medidas en el centro de Madrid, el tiempo de reparar una vivienda, el tiempo de vender una vivienda. En el tiempo de reajustar este proceso, las familias pueder ser desplazadas del bartio en estas viviendas sociales. Evidentemente, en último término, cuando el conjunto de temodelación del centro terminte, estas viviendas deberán incorporarse al patrimonio público.

En cuarto lugar, hay que tomar medidas contra la creciente desdotación en el equipamiento social y colectivo del centro de Madrid. Justa. mente por estos cambios de uso y por la distinta valoración del uso del suelo a la que nos referimos en principio, se está asistiendo al proceso de traslado de colegios, traslado incluso de edificios administrativos a la periferia, simplemente para especular con el terteno público, incluso organizado por la Administración. Evidentemente, aquí habría disposiciones muy sencillas del tipo declarar no edificable todo terreno que actualmente tenga un uso de equipamiento colectivo en la ciudad, una medida nada revolucionaria.

En quinto lugar, es necesario ligar esta remolelación general del centro a una politica de transporte: prioridad al transporte público, teservar el 
automóvil para toda una serie de usos, prohibición de circulación del automóvil en amplias zonas del centro, según un sistema de horario móvil. Con un sistema de regulaciones espacio-temporales difetenciales, puede agilizarse considerablemente el sistema de circulación, con tal de que los transportes públicos reciban una prioridad y haya una política adecuada de transportes públicos basada también en la conservación de energía.

En este sentido, otro elemento es la peatonalización de grandes zonas de la ciudad. Pero no solamente como elemento decorativo para algunas horas, para los turistas de verano, etc., sino entre otras cosas para preservar la salud cardíaca de los ciudadanos; es decir, para que haya gente que ande de vez en cuando por la ciudad, sin riesgo de ser aplastado por un aluvión de coches. Peatonalizando grandes zonas de la ciudad, se aumenta la velocidad de circulación del peatón y, por consiguiente, trayectos que hoy día son de cuarenta minutos se pueden hacer de veinte a treinta minutos tranquilamente, $y$ andar ese tiempo por la mañana o por la tarde es un principio de salud pública. Un sistema combinado de transportes públicos, aparcamientos petiféricos, control de la circulación de automóviles, zonas peatonales y estímulo al uso del instrumento privilegiado del movimiento (las piernas) sería una política del centro de tipo nuevo, en que la circulación de los coches fueta sustituida por el paso de los humanos.

Una sexta medida sería la recuperación de espacios libres de uso público. El proceso de apropiación privada que ha habido de los espacios libres o el proceso de transformación de espacios libres en aparcamientos o zonas semivalladas, hace que la apertura de espacios libres en el centro no implica la demolición, sino la recuperación de toda una serie de espacios. En el caso de que sean espacios privados, se debería llevar a cabo un proceso de negociación con los órganos gestores del patrimonio público, con la Iglesia (con todo lo que eso puede presentar de conflictivo, pero también teniendo en cuenta valores morales que esta institución puede asumir) y con los terratenientes urbanos que aún conservan sus privilegios en ef centro.

En séptimo lugar, es necesaria la preservación y apertura del patrimonio artístico. Preservación desde luego ( $\mathrm{y}$ en este sentido todas las medidas de tipo «catálogo» son fundamentales), pero también uso y apertura de ese patrimonio artístico. Lo que no puede ser es que una ciudad se convierta en un museo y que la gente pase por delante de las fachadas. Es absolutamente fundamental transformar las tumbas de arte que existen en una página viva de telación con la gente y de conexión plástica de la experiencia ciudadana con la historia de la ciudad. Esto se liga muy directamente a to que es una piedra de toque básica de toda política de renovación urbana, y es el estimulo de la vida cultural festiva y asociativa de 
los centros de las ciudades. De nada sirve conservar los edificios si no se conserva el tejido social y si no se ayuda a estimulat y desartollar las actividades y las tradiciones existentes en los barrios, incluso las que, de forma nueva, la población empieza a crear.

La falta de actividades colectivas en el centro no proviene ni de un problema de circulación ni de la apatía ciudadana, sino que es un problema social impulsado tanto por las tendencias generales de la funcionalización de la vida privada, que se ha hecho en todos los países occidentales, como por las características autoritarias de la Administración en España. Ésos son los procesos que han destruido y tienden a destruir lo que hay como tejido social. Es decir, no se trata de inventar un tejido social, sino de estimular los gérmenes que existen en dicho tejido. Por ejemplo, las asociaciones de ciudadanos son importantes, pero no sólo como asociaciones reivindicativas, sino como centros impuisores de vida cultural y social, y de hecho han suscitado en los últimos años toda una serie de fiestas y verbenas. Este intento es algo que debe ser estimulado desde la Administración sin absorberlo, creando las facilidades, el caldo de cultivo para que esto se desarrolle de forma autónoma.

Los criterios señalados se relacionan directamente con la novena medida que sería el pensar en el barrio en términos de actividades, no sólo de decorado o de flujo de circulación. Se deben estimular actividades en las que se sepa que producen un efecto de desarrollo de vida de barrio. Por ejemplo, se sabe que van desapareciendo toda una serie de bares, comercios, actividades artesanales que, efectivamente, no son tentables económicamente. La propuesta es que, en lugar de como se está haciendo ahora en algunas ciudades, por ejemplo en el bartio de Marais, en París, en el que se crea como apéndice del Ministerio de Cultura el taller attesanal que rememora el siglo $\mathrm{xxX}$ como elemento de museo, puesto que hay talleres artesanales del siglo xrx, se podrían adoptar una serie de medidas fiscales, por ejemplo, de desgravación de impuestos, para beneficiar a unos señores que económicamente no son rentables, pero que socialmente tienen el efecto de estimular una vida en torno a su trabajo. Lo mismo puede decirse con los bares existentes, con las tabernas, etc. Es decir, se requiere una politica algo más imaginativa en términos de actuar sobre el tejido social de los centros antes de que se acabe de descomponer.

La última medida sería la multinucleación de la centralidad urbana. Un centro único en una átea metropolitana es un centro que se descompone, que se satura por muchas medidas de desconcentración de actividades que se tomen. Si la única manera de tener un entorno urbano agradable el sábado por la noche es venir al centro de Madrid, ese centro de Madrid acabará haciéndose absolutamente inviable. Sólo cuando hay una jerarquía 
de centros en que, a partir de una centralidad urbana hay centros de barrio, de distrito, y hay una de esas actividades, apoyándose en el tejido urbano social existente, sólo en ese caso puede existir un centto que, a nivel de área metropolitana, sea el centro de nivel más elevado, apoyado en sus funciones por otros centros de nivel más bajo. Tales son las perspectivas de política urbana que pueden ir haciendo posible una nueva ciudad y una nueva vida.

\section{Conclusiones}

La centralidad resume las tendencias de organización y desarrollo de una ciudad. Una ciudad se expresa fundamentalmente por medio de su centro, y una política urbana va a ser resumida y articulada en términos de piedra de toque con respecto a lo que sea una política urbanística de centralismo. Para esquematizar las opciones que se plantean, de hecho no hay demasiadas alternativas con respecto a una política del centro. Sólo hay tres centros posibles en una sociedad como la nuestra.

En primer lugar, lo que puede llamarse el centro direccional funcional con islas de consumo cultural, tipo grandes ciudades norteamericanas; es decir, el centro como conglomerado de actividades administrativas, tanto públicas como privadas, con algunas zonas de consumo, tanto cultural como comercial. Este tipo de centro representa la tendencia «espontánea» de la estructura urbana que estamos viviendo, y significa, de hecho, el fin de la ciudad como mecanismo de intercambio social. Pasa a ser la punta extrema del funcionalismo urbano en el sentido de que el centro es simplemente la placa que gira en torno a una sexie de procesos. La relación de inter cambio social pasa a ser totalmente privatizada, en un cierto sentido desin. tegrada, porque esa privatización se traduce en los dos grandes instrumentos del tiempo y espacio totalmente individaalizados y sin intercambios, que son la televisión y el coche.

Otra posibilidad, que es la que «está de modà en el urbanismo cuitural y en la élite urbanística europea y norteamericana, es el centro de tipo cultural, el centro ligado a la organización de un consumo cultural de élite. Tal es, por ejemplo, lo que se realiza en París, en el Marais y en Les Halles; o lo que trata de estimularse en algunas ciudades norteamericanas; $o$, en cierto sentido, lo que en algunos momentos semiespontáneamente en Barcelona se ha intentado hacer en torno a una revitalización del bartio gótico conectado con las Ramblas. Tal centro es la expresión del nuevo espacio de la élite. En efecto, toda sociedad se caracteriza porque su clase dominante se construye un espacio. El espacio tipo autopista y Central 
Business District ya no es el espacio de la élite. Las élites de nuestra sociedad son aquellas que organizan su espacio de forma que su residencia, su trabajo y su ocio se realicen en el mismo barrio, y, por tanto, pueden des. plazarse a pie: sólo salen de ese espacio reducido para trasladarse de continente o de país, y desde luego en avión. En el fondo es el espacio de la élite cultural, política y económica francesa, norteameticana, inglesa. Ese tipo de centro expresa una profunda desigualdad social, lo que podría llamarse el aristocratismo urbano: recuperar la ciudad, pero para la élite. Ahora bien, con un cierto grado de cinismo, puede decirse que ese centro podría ser atractivo, por lo menos, para los medios dirigentes. Pero, al mismo tiempo, el disfrute de ese centro se bace imposible en la práctica. ¿Por qué? Porque todavía no se pueden plantar alambres electrificados en torno a ese centro, y como la sociedad en la que vivimos es una fuente permanente de frustración, ese centro sólo se mantiene en buenas condiciones, como centro cultural bien pintado, bien organizado, con sus restaurantes simpáticos, centros culturales, etc., durante un breve lapso de tiempo. Porque al cabo de ese tiempo se conviette en la mezcla de esa élite con la expresión violenta, brutal y abierta de la marginalidad social.

La razón de tal proceso reside en la artificialidad de esa centralidad urbana: al no descansar sobre ningún tejido social de base de ese trozo de ciudad, sino al ser más bien un decorado que se planta de manera voluntarista, ese decorado se ve invadido por lo que es justamente la masa flotante de nuestra sociedad, es decir, los desarraigados de todo tipo. Ésa ha sido la experiencia en el Barrio Latino de París, en las Ramblas de Barcelona, en Greenwich Village de Nueva York, o en Georgetown en Washington; y está empezando a ser confusamente la experiencia de Malasaña, en Madrid. El problema en ese sentido es que ese centro se construye como centro de élite, que después se lo apropia la marginalicład social, lo que en cierto sentido podría ser un factor estimulante, pero entonces es absolutamente reprimido, se convierte en un centro invadido no por la élite ni por la marginalidad, sino por la policía. Tal es el destino de la mayoría de esos centros artificiales de tipo cultural que no reposan sobre un tejido social construido en la residencia y la actividad cotidianas.

El tercer tipo de centro es un centro formado por la revitalización del tejido social urbano existente, o al menos existente en principio, en el que, por tanto, hay un medio cultural y un entorno que existe, y que sin tener que reprimir Ia marginalidad integra su invasión, y sin tener que excluir a la élite, no es aptopiado por la élite. En Ia medida en que se da una vitalidad urbana y social propia de ese centro, en la medida en que, por otro lado, hay una multicentralidad, es decir, que no es un centro único sino que se remite a una serie de centros de niveles, jerarquías y usos 
diferentes, se pernite entonces, por un lado, asegurar el intercambio entre las distintas esferas y entre los distintos usos sociales; por otro lado, respetar la diversidad de la sociedad; y por otro lado, diluir la marginalidad social o especializarla, en lugar de ser sumergido por esta marginalidad y convertirla no en la sal, sino en la pimienta de nuestra sociedad. Y es evidente que la pimienta necesita un guiso social en el que pueda disolverse para, al mismo tiempo, ser digerible y dar sabor.

Ese tipo de centro, que responde a las proposiciones anteriormente expuestas, requiere una serie de condiciones que mencionaremos esquemáticamente. Requiere una nueva política urbana que ligue eficacia técnica, imaginación urbanística y capacidad administrativa. Requiere también unas nuevas condiciones políticas que pasan necesariamente por una capacidad de apertura de la Administración urbanística a la sociedad, por una capacidad de sensibilidad de la Administración a la expresión, por un Iado, de las asociaciones ciudadanas; y por otro, a la sensibilidad de lo que pasa en una ciudad. Lo cual implica un cambio político en la Administración municipal existente.

Pero, de todas formas, en distintas situaciones políticas, una política de centralidad urbana de este tipo se presenta, a la vez, como una alternativa posible y como una exigencia cultural para que nuestras ciudades se expresen en nuestros centros y para que nuestros centros sean la expresión de nuestros deseos, nuestros proyectos, nuestras voluntades y nuestros sueños.

\section{Manuel Castelles}

Centre d'Etude des Mouvements Sociaux

53 Boulevard Raspail

75006 Paris

Francia 